\title{
SPACES WITHOUT COHOMOLOGICAL DIMENSION PRESERVING COMPACTIFICATIONS
}

\author{
JERZY DYDAK AND JOHN J. WALSH
}

(Communicated by James E. West)

\begin{abstract}
Examples are constructed that include: first, a separable metric space having cohomological dimension $\mathbf{4}$ such that every Hausdorff compactification has cohomological dimension at least 5 ; second, a locally compact metric space having cohomological dimension 4 whose Stone-Čech compactification has infinite cohomological dimension.
\end{abstract}

\section{INTRODUCTION}

This note presents examples that illustrate the distinctly different behavior that compactifications can exhibit in the setting of cohomological dimension in contrast to the setting of covering dimension.

While a space $X$ and its Stone-Čech compactification $\beta(X)$ have the same covering dimension (a fact that follows easily from the extension property that characterizes $\beta(X)$ and the characterization of dimension stated later in this section), A. N. Dranishnikov produced examples, for each $n \geq 4$, of locally compact separable metric spaces having integral cohomological dimension $n$ whose Stone-Čech compactifications have integral cohomological dimension at least $n+1\left[\mathrm{Dr}_{2}\right]$. The first class of examples constructed below sharpens the conclusion as it consists of locally compact separable metric spaces having finite integral cohomological dimension whose Stone-Čech compactifications have infinite integral cohomological dimension.

A separable metric space embeds in a compact metric space having the same covering dimension [HW, p. 65] and, consequently, has a metric compactification having the same covering dimension. In contrast, the second class of examples constructed below includes, for each $n \geq 4$, a separable metric space having integral cohomological dimension $n$ such that every Hausdorff compactification has integral cohomological dimension at least $n+1$. These examples provide solutions to problems raised in [Ru] as well as to problem D19 in [We].

Received by the editors April 27, 1990.

1980 Mathematics Subject Classification (1985 Revision). Primary 55M11, 54F54.

Key words and phrases. Cohomological dimension, Eilenberg-Mac Lane complexes, compactifications, complex $k$-theory.

The second author was supported in part by a grant from the National Science Foundation. 
The construction of the examples makes extensive use of the technique in $\left[\mathrm{Dr}_{1}\right]$ that is used to identify compact metric spaces that have finite cohomological dimension but are, in fact, infinite dimensional. Specifics of that construction are not needed and the relevant properties are listed as needed.

Recall that a space $X$ is said to have integral cohomological dimension $\leq n$, written $\operatorname{dim}_{\mathbf{Z}} X \leq n$, provided, for every map $f: A \rightarrow K(\mathbf{Z}, n)$ of a closed subset $A \subset X$ to an Eilenberg-Mac Lane complex $K(\mathbf{Z}, n)$, there is an extension to a map $F: X \rightarrow K(\mathbf{Z}, n)$. Evidently, this is a "classifying space" statement that the inclusion-induced homomorphism of Čech cohomology $H^{n}(X ; \mathbf{Z}) \rightarrow H^{n}(A ; \mathbf{Z})$ is onto for every closed subset $A \subset X$ and is, in fact, equivalent to requiring that $H^{q}(A, B ; \mathbf{Z})=0$ for all closed pairs $B \subset A \subset X$ and all $q \geq n+1$. (In the setting of metric spaces or of compact Hausdorff spaces, which suffice for our purposes, simply requiring that the Eilenberg-Mac Lane spaces be chosen to be CW-complexes leads to a "coherent" theory. There are additional complications in the more general settings of paracompact spaces or of normal spaces.)

The above definition of cohomological dimension compares nicely with the following characterization of covering dimension: a space $X$ has covering dimension $\leq n$, written $\operatorname{dim} X \leq n$, provided, for every map $f: A \rightarrow S^{n}$ of a closed subset $A \subset X$ to an $n$-sphere, there is an extension to a map $F: X \rightarrow S^{n}$.

Following standards conventions, $\operatorname{dim}_{\mathrm{z}} X$ is set equal to the least integer $n$ such that $\operatorname{dim}_{\mathrm{z}} X \leq n$ and is set equal to $\infty$ if $\operatorname{dim}_{\mathrm{z}} X \pm n$ for all $n$.

First class of examples. For each $n \geq 4$, there is a locally compact metrizable space $X$ with $\operatorname{dim}_{\mathrm{z}} X \leq n$ and $\operatorname{dim}_{\mathrm{z}} \beta(X)=\infty$, where $\beta(X)$ is the Stone-Čech compactification of $X$.

Second class of examples. For each $n \geq 4$, there is a metrizable space $X$ with $\operatorname{dim}_{\mathrm{z}} X \leq n$ such that, for every compact Hausdorff space $Y$ with $X \hookrightarrow Y$, $\operatorname{dim}_{\mathrm{z}} Y \geq n+1$.

\section{Preliminaries: Notations ANd CONVEntions}

Throughout the paper the Eilenberg-Mac Lane spaces $K(\mathbf{Z}, n)$ are assumed to have a CW-structure such that each $m$-skeleton $K(\mathbf{Z}, n)^{(m)}$ is a finite complex and $K(\mathbf{Z}, n)^{(n+1)}=K(\mathbf{Z}, n)^{(n)}=S^{n}$.

The reduced complex k-theory of a space $X$ is denoted by $k^{*}(X)=k^{0}(X) \oplus$ $k^{1}(X)$ where $k^{1}(X)=[X, U], U$ being the unitary group, and $k^{0}(X)=$ $[X, B U], B U$ being the classifying space of the group $U$. Namely, $B U$ is the increasing union of $G_{1}\left(\mathbf{C}^{2}\right) \subset G_{2}\left(\mathbf{C}^{4}\right) \subset \cdots \subset G_{n}\left(\mathbf{C}^{2 n}\right) \subset \cdots$, where $G_{m}\left(\mathbf{C}^{n}\right)$ is the Grassmann manifold consisting of $m$-dimensional subspaces of $\mathbf{C}^{n}$. The reduced k-theory with $\mathbf{Z}_{p}$-coefficients is denoted by $k^{*}\left(X ; \mathbf{Z}_{p}\right)$. Formally, $k^{0}\left(X ; \mathbf{Z}_{p}\right)=\left[X \wedge M_{p}, B U\right]=\left[X, B U^{M_{p}}\right]$ and $k^{1}\left(X ; \mathbf{Z}_{p}\right)=$ $\left[X \wedge M_{p}, U\right]=\left[X, U^{M_{p}}\right]$, where $M_{p}$ is the Moore space obtained by attaching a $B^{2}$ to $S^{1}$ by a degree $p$ map $f: S^{1} \rightarrow S^{1}$. Our sources for computations of 
the k-theory of Eilenberg-Mac Lane spaces are $[\mathrm{AH}]$ and [BM].

The following are the "formal" ingredients that are needed to put together the promised examples. The first of these is the central result in $\left[\mathrm{Dr}_{1}\right]$ and the reader is referred there for an exposition, the focus of that paper being that, choosing the $P$ in Fact 1 below to be a sphere of dimension $>q$, the space $X$ must be infinite dimensional. The extraction of the remaining three, which uses computations of the k-theory of Eilenberg-Mac Lane spaces, is presented in $\S \S 4-6$. The first class of examples are constructed using Facts 1 and 2 and the second class using Facts 1 and 4 . Fact 3 is needed in establishing Fact 4.

Fact 1. Given an integer $q \geq 3$, a compact polyhedron $P$, a prime $p$, and a nonzero element $\alpha \in k^{*}\left(P ; \mathbf{Z}_{p}\right)$, there is compactum $X$ with $\operatorname{dim}_{\mathbf{Z}} X \leq q$ and a map $f: X \rightarrow P$ with $f^{*}(\alpha) \neq 0$ in $k^{*}\left(X ; \mathbf{Z}_{p}\right)$.

Fact 2. For $s \geq 3$ and $K=K(\mathbf{Z}, s)$, there is an integer $m(s) \geq s+1$ such that, for every $q \geq m(s)$, there is a prime $p$ such that the inclusion-induced homomorphism $k^{*}\left(K^{(q)} ; \mathbf{Z}_{p}\right) \rightarrow k^{*}\left(K^{(m(s))} ; \mathbf{Z}_{p}\right)$ is not trivial.

The next two facts are refinements of Fact 2. While the latter is a formal consequence of the k-theory of $K(\mathbf{Z}, s)$ being nonzero but purely "phantom," these require more exacting computations.

Fact 3. For $s \geq 0, K=K(\mathbf{Z}, 2 s+1)$, and $m \geq s+1$, the inclusion-induced homomorphism $k^{1}\left(K^{(2 m)} ; \mathbf{Z}_{p}\right) \rightarrow k^{1}\left(S^{2 s+1} ; \mathbf{Z}_{p}\right)$ is an monomorphism for all but finitely many primes $p$.

Fact 4. For $s \geq 1, K=K(\mathbf{Z}, s)$, and $2 m \geq s$, the inclusion-induced homomorphism $k^{*}\left(K^{(2 m)} ; \mathbf{Z}_{p}\right) \rightarrow k^{*}\left(S^{s} ; \mathbf{Z}_{p}\right)=\mathbf{Z}_{p}$ is an epimorphism for all but finitely many primes $p$.

\section{CONSTRUCTION OF FIRST CLASS OF EXAMPLES}

Fix an integer $n \geq 4$. For each $s \geq n$, let $m(s) \geq n+1$ be as specified in Fact 2. Although, for any space $X$ with $\operatorname{dim}_{\mathbf{z}} X \leq n-1$, every map $f: X \rightarrow$ $K(\mathbf{Z}, s)$ is null-homotopic, the next claim records that such null-homotopies may require the use of a "large" dimensional skeleton of $K(\mathbf{Z}, s)$.

Claim. For each $q \geq m(s)$, there is a compactum $Y_{s, q}$ with $\operatorname{dim}_{\mathrm{Z}} Y_{s, q} \leq n-1$ and a map $f_{s, q}: Y_{s, q} \rightarrow K(\mathbf{Z}, s)^{(m(s))}$ such that $f_{s, q}$ is essential as a map into $K(\mathbf{Z}, s)^{(q)} \supset K(\mathbf{Z}, s)^{(m(s))}$.

Proof of Claim. Using Fact 2 for $K=K(\mathbf{Z}, s)$, specify a prime $p$ and a nonzero element $\alpha \in$ image of $k^{*}\left(K^{(q)} ; \mathbf{Z}_{p}\right)$ in $k^{*}\left(K^{(m(s))} ; \mathbf{Z}_{p}\right)$. Using Fact 1 , specify a compact space $Y_{s, q}$ with $\operatorname{dim}_{\mathrm{Z}} Y_{s, q} \leq n-1$ and a map $f_{s, q}: Y_{s, q} \rightarrow$ $K(\mathbf{Z}, s)^{(m(s))}$ such that $f_{s, q}^{*}(\alpha) \neq 0$ in $k^{*}\left(Y_{s, q} ; \mathbf{Z}_{p}\right)$. The choice of $\alpha$ detects that $f_{s, q}$ is essential as a map into $K(\mathbf{Z}, s)^{(q)}$. 
Set $X$ equal to the disjoint union of the cones, say $c\left(Y_{s, q}\right)$, for $s \geq n$ and $q \geq m(s)$. For each cone, $\operatorname{dim}_{\mathrm{Z}} c\left(Y_{s, q}\right) \leq \operatorname{dim}_{\mathrm{Z}} Y_{s, q}+1 \leq n$ and, hence, $\operatorname{dim}_{\mathrm{z}} X \leq n$. It remains to show that $\operatorname{dim}_{\mathrm{z}} \beta(X)=\infty$. Suppose otherwise, namely, that $\operatorname{dim}_{\mathrm{Z}} \beta(X) \leq r$. Set $Z$ equal to the closed subset of $X$ that is the union of the various $Y_{s, q}$ 's. Define $g: Z \rightarrow K(\mathbf{Z}, r)^{(m(r))}$ by setting $\left.g\right|_{Y(s, q)}$ equal to $f_{r, q}$ for $s=r$ and equal to a constant function for $s \neq r$. Now $g$ has an extension to $\tilde{g}: \beta(Z) \rightarrow K(\mathbf{Z}, r)^{(m(r))}$, as the latter is compact. Since $\beta(Z) \subset \beta(X)$ is a closed subset and $\operatorname{dim}_{\mathrm{z}} \beta(X) \leq r, \quad \tilde{g}$ extends to a map $G: \beta(X) \rightarrow K(Z, r)$. Since the image of $G$ is compact, it is contained in $K(\mathbf{Z}, r)^{(t)}$ for some $t$. In particular, for any $q \geq \operatorname{maximum}\{t, m(s)\}$, the restriction of $G$ to $c(Y(r, q))$ is a null-homotopy of $f_{r, q}$ in $K(\mathbf{Z}, r)^{(q)}$, contradicting the conclusion recorded in the above claim.

\section{CONSTRUCTION OF SECOND CLASS OF EXAMPLES}

Fix an integer $n \geq 4$ and set $K=K(\mathbf{Z}, n)$. Using Fact 4 , for any $m \geq$ $n+1$, there is a prime $p_{m}$ such that the inclusion-induced homomorphism $k^{*}\left(K^{(2 m)} ; \mathbf{Z}_{p_{m}}\right) \rightarrow k^{*}\left(S^{n} ; \mathbf{Z}_{p_{m}}\right)$ has non trivial image. Name an element $\alpha_{m} \neq 0$ in the image. Using Fact 1, there is a compactum $X_{m}$ with $\operatorname{dim}_{\mathbf{Z}} X_{m} \leq n-1$ and a map $f_{m}: X_{m} \rightarrow S^{n}$ such that the image of $\alpha_{m}$ in $k^{*}\left(X_{m} ; \mathbf{Z}_{p_{m}}\right)$ is not zero. Notice that the map $f_{m}$ is essential as a map into $K^{(2 m)}$.

Set $c\left(f_{m}\right)$ equal to the mapping cone of $f_{m}$. Namely, setting

$$
M\left(f_{m}\right)=X_{m} \times[0,1] \cup_{f_{m}} S^{n} \text { and } c\left(X_{m}\right)=X_{m} \times[-1,0] / X_{m} \times\{-1\}
$$

where $f_{m}: X_{m} \times\{1\} \rightarrow S^{n}$ is defined by setting $f_{m}(x, 1)=f_{m}(x)$,

$$
c\left(f_{m}\right)=c\left(X_{m}\right) \cup_{X_{m} \times\{0\}} M\left(f_{m}\right) .
$$

Noting that each $c\left(f_{m}\right)$ contains a "natural" copy of $S^{n}$, set $X$ equal to the union of the $c\left(f_{m}\right)$ 's, for $m \geq n+1$, with these $n$-spheres identified to each other. That is, $c\left(f_{m}\right) \subset X$ and $c\left(f_{m}\right) \cap c\left(f_{m^{\prime}}\right)=S^{n}$ for $m \neq m^{\prime}$. Topologize $X$ with the "natural" metric topology (the quotient topology is not metrizable) that makes each inclusion $c\left(f_{m}\right) \hookrightarrow X$ an embedding.

Suppose that $X \hookrightarrow Y$ for a compact Hausdorff space $Y$ with $\operatorname{dim}_{\mathrm{z}} Y \leq$ $n$. Then the inclusion $S^{n} \hookrightarrow K(Z, n)$ (of the sphere sitting in each $c\left(f_{m}\right)$ ) extends to a map $h: Y \rightarrow K(\mathbf{Z}, n)$. Since $h(Y)$ is compact, it is contained in $K(\mathbf{Z}, n)^{(m)}$, for some $m \geq n+1$. The restriction of $h$ to $c\left(f_{m}\right)$ yields a null-homotopy of $f_{m}$ in $K(\mathbf{Z}, n)^{(m)}$, contradicting that $f_{m}$ is essential as a map into $K(\mathbf{Z}, n)^{(2 m)}$.

\section{Proof of FaCt 2}

For any generalized cohomology theory, there is a short exact sequence, due to Milnor [Mi], that measures the cohomology of an infinite complex in terms of the cohomology of its finite subcomplexes. In the current context, recalling 
the standing assumption that the Eilenberg-Mac Lane complexes are chosen to have finite skeleton in each dimension, there is a short exact sequence

$$
0 \rightarrow \lim ^{1}\left\{\left(k^{*}\left(K^{(m)}\right)\right\} \rightarrow k^{*}(K) \rightarrow \lim _{\longleftarrow}\left\{k^{*}\left(K^{(m)}\right)\right\} \rightarrow 0 .\right.
$$

By computations in $[\mathrm{AH}]$ and $[\mathrm{BM}]$, the inverse limit $\lim \left\{k^{*}\left(K^{(m)}\right)\right\}=0$ but $k^{*}(K) \neq 0$. Consequently, $\lim ^{1}\left\{\left(k^{*}\left(K^{(m)}\right)\right\} \neq 0\right.$. In particular, there must be a $m(s) \geq n+1$ such that the image of $i^{*}: k^{*}\left(K^{(q)}\right) \rightarrow k^{*}\left(K^{m(s)}\right)$ is infinite for $q \geq m(s)$. The skeleton being finite assures that $k^{*}\left(K^{m(s)}\right)$ is finitely generated. If $p$ is a prime such that the cokernel of $i^{*}$ has no $p$-torsion, then the image of $k^{*}\left(K^{(q)}\right) \otimes \mathbf{Z}_{p} \rightarrow k^{*}\left(K^{m(s)}\right) \otimes \mathbf{Z}_{p}$ is not trivial. In turn, the universal coefficient theorem (for example, see [AH, $\S 1]$ ) reveals that the homomorphism $k^{*}\left(K^{(q)} ; \mathbf{Z}_{p}\right) \rightarrow k^{*}\left(K^{(m(s))} ; \mathbf{Z}_{p}\right)$ is not trivial.

\section{Proof of FACT 3}

Set $K=K(\mathbf{Z}, 2 n+1)$ and recall that $H^{q}(K, \mathbf{Q})=0$ for $q \geq 2 n+2$; see [FFG, p.174]. (The latter reference contains serious "translation errors," one occurring in the first line on p.174 where the phrase "finitely generated" should be "finite." Nevertheless, the proofs are reasonably accessible.)

The first step is to exploit the vanishing of the higher rational cohomology groups to detect that the groups $H^{q}\left(K ; \mathbf{Z}_{p}\right)=0,2 n+2 \leq q \leq 2 m+1$, for all but finitely many primes $p$. Noting that the groups $H^{q}(K ; \mathbf{Z})$ are finitely generated, these groups must be torsion groups for $q \geq 2 n+2$ (otherwise a universal coefficient theorem detects that $\left.H^{q}(K ; \mathbf{Q}) \neq 0\right)$. Setting $\mathscr{P}$ equal to the set of primes $p$ such that there is no $p$-torsion in $H^{q}(K ; \mathbf{Z})$, for $2 n+2 \leq$ $q \leq 2 m+1, \mathscr{P}$ consists of all but finitely many primes and $H^{q}\left(K ; \mathbf{Z}_{p}\right)=0$, for $p \in \mathscr{P}$ and $2 n+2 \leq q \leq 2 m+1$.

Set $L$ equal to the quotient $K^{(2 m)} / S^{2 n+1}$. Using the portion of the long exact sequence

$$
k^{1}\left(L ; \mathbf{Z}_{p}\right) \rightarrow k^{1}\left(K^{(2 m)} ; \mathbf{Z}_{p}\right) \rightarrow k^{1}\left(S^{2 n+1} ; \mathbf{Z}_{p}\right),
$$

it suffices to establish that $k^{1}\left(L ; \mathbf{Z}_{p}\right)=0$ for each $p \in \mathscr{P}$.

Fix a prime $p \in \mathscr{P}$ and let $\left\{\rightarrow \cdots \rightarrow E_{q+1} \rightarrow E_{q} \rightarrow \cdots \rightarrow E_{0}=\{\right.$ point $\left.\}\right\}$ be the Postnikov system of $U^{M_{p}}$. Namely, $E_{q+1} \rightarrow E_{q}$ is a fibration with fiber $K\left(\pi_{q+1}\left(U^{M_{p}}\right), q+1\right)$ and $\pi_{k}\left(E_{q}\right)=0$ for $k>q$. The representation $k^{1}\left(L ; Z_{p}\right)=\left[L, U^{M_{p}}\right]$ and the complex $L$ having dimension $2 m$ reduce the problem to showing that $\left[L, E_{2 m+1}\right]=0$. Let

$$
s=\max \left\{q: q \leq 2 m+1 \text { and }\left[L, E_{q}\right]=0\right\} .
$$

Since $L$ has no cells having dimension between 0 and $2 n+3,\left[L, E_{q}\right]=0$ for $q \leq 2 n+2$. Hence, $s \geq 2 n+2$. Suppose that $s<2 m+1$. The even-dimensional homotopy groups of $U^{M_{p}}$ are trivial and the odd-dimensional ones are $\mathbf{Z}_{p}$. In particular, $E_{q+1} \rightarrow E_{q}$ is a homotopy equivalence for $q$ odd and, hence, $s$ must be even. 
Since $s \geq 2 n+2$, the exact sequence

$$
H^{s+2}\left(S^{2 n+1} ; \mathbf{Z}_{p}\right) \rightarrow H^{s+1}\left(L ; \mathbf{Z}_{p}\right) \rightarrow H^{s+1}\left(K^{(2 m)} ; \mathbf{Z}_{p}\right) \rightarrow H^{s+1}\left(S^{2 n+1} ; \mathbf{Z}_{p}\right)
$$

reveals an isomorphism $H^{s+1}\left(L ; \mathbf{Z}_{p}\right) \simeq H^{s+1}\left(K^{(2 m)} ; \mathbf{Z}_{p}\right)$. However, the latter group is trivial: either $s+1=2 m+1$ and $H^{s+1}\left(K^{(2 m)} ; \mathbf{Z}_{p}\right)=0$ due to dimension considerations, or $s+1<2 m$ (recall that $s$ is even) and $H^{s+1}\left(K^{(2 m)} ; \mathbf{Z}_{p}\right)=$ $H^{s+1}\left(K ; \mathbf{Z}_{p}\right)=0$ as $s+1 \geq 2 n+2$.

Any map $L \rightarrow E_{s+1}$ is homotopic to a map into the fiber, i.e., a $K\left(\mathbf{Z}_{p}, s+1\right)$. The representation $\left[L, K\left(\mathbf{Z}_{p}, s+1\right)\right]=H^{s+1}\left(L ; \mathbf{Z}_{p}\right)$ and the triviality of the latter group detects that maps of $L$ into the fiber are null-homotopic. Hence, $\left[L, E_{s+1}\right]=0$. In particular, the supposition that $s<2 m+1$ cannot be valid and, thus, $s=2 m+1$ and the proof is completed.

\section{Proof of FACT 4}

Set $K=K(\mathbf{Z}, n)$. The proof differs depending on whether $n$ is even or odd. Recall that for $n$ odd $k^{0}\left(S^{n} ; \mathbf{Z}_{p}\right)=0$ and $k^{1}\left(S^{n} ; \mathbf{Z}_{p}\right)=\mathbf{Z}_{p}$, while for $n$ even $k^{0}\left(S^{n} ; \mathbf{Z}_{p}\right)=\mathbf{Z}_{p}$ and $k^{1}\left(S^{n} ; \mathbf{Z}_{p}\right)=0$.

Case that $n$ is odd. The case that $n=1$ is clear so assume that $n \geq 3$. There is the short exact sequence due to Milnor [Mi]

$$
0 \rightarrow \lim ^{1}\left\{k^{1}\left(K^{(m)}\right)\right\} \rightarrow k^{0}(K) \rightarrow \lim \left\{k^{0}\left(K^{(m)}\right\} \rightarrow 0 .\right.
$$

The computations in $[\mathrm{AH}]$ are that $\lim \left\{k^{0}\left(K^{(m)}\right\}=0\right.$ but $k^{0}(K) \neq 0$. Consequently, the term $\lim ^{1}\left\{k^{1}\left(K^{(m)}\right)\right\} \neq 0$. Hence, there must exist $m \geq n+1$ such that the image of $k^{1}\left(K^{(q)}\right)$ in $k^{1}\left(K^{(2 m)}\right)$ is infinite for all $q$ sufficiently large. Fix one such $q$. Exactly as in the proof in $\S 4$, conclude that for all but finitely many primes $p$, the image of $k^{1}\left(K^{(q)} ; \mathbf{Z}_{p}\right)$ in $k^{1}\left(K^{(2 m)} ; \mathbf{Z}_{p}\right)$ is not trivial. Since the latter group injects into $k^{1}\left(S^{n} ; \mathbf{Z}_{p}\right)=\mathbf{Z}_{p}$ for all but finitely many primes (by Fact 3 ), the conclusion that $k^{1}\left(K^{(2 m)} ; \mathbf{Z}_{p}\right) \rightarrow k^{1}\left(S^{n} ; \mathbf{Z}_{p}\right)$ is an epimorphism for all but finitely many primes $p$ follows.

Case that $n$ is even. Set $L=K^{(2 m)} / S^{n}$. The exact sequence

$$
k^{0}\left(K^{(2 m)} ; \mathbf{Z}_{p}\right) \rightarrow k^{0}\left(S^{n} ; \mathbf{Z}_{p}\right) \rightarrow k^{1}\left(L ; \mathbf{Z}_{p}\right)
$$

reveals that it suffices to show that $k^{1}\left(L ; \mathbf{Z}_{p}\right)=0$ for all but finitely many primes. The proof is similar to that in $\S 5$.

The group $H^{2 q+1}(K ; \mathbf{Q})=0$ for all $q$, see [FFG, p. 174]. Setting $\mathscr{P}$ equal to the set of primes $p$ such that $H^{2 q+1}\left(K ; \mathbf{Z}_{p}\right)=0$ for $q \leq 2 m, \mathscr{P}$ consists of all but finitely many primes. In view of the representation $k^{1}\left(L ; \mathbf{Z}_{p}\right)=$ $\left[L, U^{M_{p}}\right]$, it remains to show that $\left[L, U^{M_{p}}\right]=0$ for all $p \in \mathscr{P}$. 
Fix a prime $p \in \mathscr{P}$ and let $\left\{\rightarrow \cdots \rightarrow E_{q+1} \rightarrow E_{q} \rightarrow \cdots \rightarrow E_{0}=\{\right.$ point $\left.\}\right\}$ be the Postnikov system of $U^{M_{p}}$. Since $L$ has dimension $2 m$, it suffices to show that $\left[L, E_{2 m+1}\right]=0$. Set $s=\max \left\{q: q \leq 2 m+1\right.$ and $\left.\left[L, E_{q}\right]=0\right\}$. Since $L$ has no cells having dimension between 0 and $n+2,\left[L, E_{q}\right]=0$ for $q \leq n+1$. Hence, $s \geq n+1$. Suppose that $s<2 m+1$. The even-dimensional homotopy groups of $U^{M_{p}}$ are trivial and the odd-dimensional ones are $\mathbf{Z}_{p}$. Hence, $E_{q+1} \rightarrow E_{q}$ is a homotopy equivalence for $q$ odd and, hence, $s$ must be even.

As in the proof in $\S 5$, there is an isomorphism

$$
H^{s+1}\left(L ; \mathbf{Z}_{p}\right) \simeq H^{s+1}\left(K^{(2 m)} ; \mathbf{Z}_{p}\right) .
$$

Either $s+1=2 m+1$ and the latter group is trivial for dimension considerations or $s+1<2 m$ and the group is trivial since it is isomorphic to $H^{s+1}\left(K ; \mathbf{Z}_{p}\right)$, which is trivial since $s+1$ is odd. Exactly as in the proof in $\S 5$, the contradiction is reached that each map $L \rightarrow E_{s+1}$ is null-homotopic and, thus, $s=2 m+1$.

\section{FinAL COMMENTS}

A. N. Dranishnikov suggested an alternate approach that quickly provides a version of Fact 4 that is adequate for the construction in $\S 3$. Fact 4 is a consequence of the inclusion-induced homomorphism $k^{*}\left(K^{(m)}\right) \rightarrow k^{*}\left(S^{S}\right)$ being nontrivial for $m \geq s$. The latter is a consequence of the commutative diagram, where $K=K^{(m)}$,

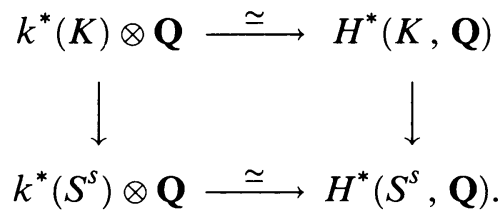

The horizontal isomorphisms are induced by the Chern character. Evidently, the right-hand vertical homomorphism is not trivial and, hence, neither is the lefthand vertical homomorphism. In turn, the homomorphism $k^{*}(K) \rightarrow k^{*}\left(S^{s}\right)$ must be nontrivial.

\section{REFERENCES}

[AH] D. W. Anderson and L. Hodgkin, The K-theory of Eilenberg-Mac Lane complexes, Topology 7 (1968), 317-329.

[BM] V. M. Buchstaber and A. S. Mischenko, $K$-theory on the category of infinite $C W$ complexes, Izv. Akad. Nauk Mat Ser. 32 (1968), 560-604.

[Dr ${ }_{1}$ ] A. N. Dranishnikov, On a problem of P. S. Alexandroff, Mat. Sb. (N.S.) 135 (1988), 551-557. (Russian)

$\left[\mathrm{Dr}_{2}\right]+$, Cohomological dimension is not preserved by Stone-Čech compactification, C. $\mathbf{R}$. Acad. Bulgare Sci. 41 (1988), 9-10. (Russian)

[FFG] A. T. Fomenko, D. B. Fuchs, and V. L. Gutenmacher, Homotopic topology, Akadémia Kiadó, Budapest, 1986. 
[HW] W. Hurewicz and H. Wallman, Dimension theory, Princeton Univ. Press, Princeton, NJ, 1948.

[Ku] W. I Kuzminov, Homological dimension theory, Russian Math. Surveys 23 (1968), 1-45.

[Mi] J. W. Milnor, On axiomatic homology theory, Pacific J. Math. 12 (1962), 337-341.

[Ru] L. R. Rubin, Characterizing cohomological dimension: the cohomological dimension of $A \cup B$, preprint.

[We ] J. West, Open problems in infinite dimensional topology, Open Problems in Topology, (J. van Mill and G. M. Reed, eds.), North-Holland, Amsterdam, pp. 523-597.

Department of Mathematics, University of Tennessee, Knoxville, Tennessee 37996 E-mail address: dydak@utkvx.bitnet

Department of Mathematics, University of California, Riverside, California 92521

E-mail address: walsh@ucrmath.ucr.edu 